\title{
Conditioned enhancement as a function of the percentage of CS-US pairings and CS duration
}

\author{
DONALD MELTZER and ROBERT J. HAMM \\ Southern Ilinois University, Carbondale, Illinois 62901
}

\begin{abstract}
Eighteen rats were trained to barpress on a variable interval 90-sec schedule of sucrose-solution reinforcement. Ss were then divided into three groups $(N=6)$ and exposed to a classical conditioning contingency while barpressing, in which a 2-min light CS preceded the delivery of a food pellet US on either $100 \%, 66 \%$, or $33 \%$ of the CS presentations. When the response rate during the CS was compared to response rate prior to the CS, it was found that the CS response rate was directly related to the percentage of US presentation. When the CS duration was changed to $8 \mathrm{sec}$, the highest CS response rates were associated with the partial pairing of CS and US.
\end{abstract}

Since Estes and Skinner (1941) first described the procedure for producing a conditioned emotional response in rats, a number of other Es have examined the parameters affecting this phenomenon. One of those parameters has been the probability that the electric shock which is usually the unconditioned stimulus (US) will follow the conditioned stimulus (CS). Geller (1964) and Wagner, Siegel, and Fein (1967, Experiment II) reported no significant differences in suppression between groups in which the US followed CS $100 \%$ of the time as opposed to groups in which the US followed the CS 50\% of the time. Brimer and Dockrill (1966) found the rate of acquisition of response suppression to be faster for a $100 \%$ group than for either a $50 \%$ or $25 \%$ group. However, all groups showed complete response suppression as conditioning proceeded, and there was no significant difference between groups at asymptotic levels of conditioning. Willis (1969) also reported that the percentage of reinforcement had no effect on response suppression. He found that when the shock US was presented on $30 \%$ or more of the trials all groups showed almost complete suppression during the CS. Only one experiment (Willis \& Lundin, 1966) varied the probability of the shock US within Ss. In this experiment, the degree of response suppression was directly related to the probability of US presentation. The reason for the lack of correlation between degree of response suppression and US probability in all but the Willis and Lundin (1966) experiment was probably some type of "floor effect." Once response rate during the CS was zero, higher US probabilities could not increase response suppression. However, many other classically conditioned responses occur with reduced probability when the probability of US presentation is reduced. Gormezano and Moore (1969) have recently reviewed a number of experiments which show this effect.

To investigate this area further, the present experiment used a variation of the CER procedure in which a positive US, such as food, follows the CS rather than a negative US, such as shock. As might be expected, several experiments using this procedure have found that operant response rate increased during the presence of a CS which precedes a positive US such as food or water (Herrnstein \& Morse, 1957; Brady, 1961; Henton \& Brady, 1970; LoLordo, 1971). This increase in response probability during the $\mathrm{CS}$ is commonly referred to as conditioned enhancement. Using this procedure, Meltzer and Brallek (1970) found they could obtain both conditioned enhancement and suppression by varying CS duration. A long CS duration resulted in conditioned enhancement while short CS durations resulted in response suppression during the CS. Thus, by taking advantage of the effect of CS duration, it is possible to further investigate the effect of partial reinforcement in a response suppression situation and also in a response enhancement situation. To accomplish this, the present investigation manipulated the percentage of positive US presentations at both long and short CS durations. It was expected that the $100 \%$ US probability schedule would result in the greatest enhancement at long CS durations and the greatest suppression at short CS durations. Less frequent presentations of the US were expected to produce weaker enhancement at long CS durations and weaker suppression at short CS durations.

\section{METHOD}

\section{Subjects}

Ss were 18 male hooded rats between the ages of 90 and 120 days at the outset of the experiment. Ten days prior to the experiment Ss were placed and maintained on a 23-h food deprivation schedule. Water was continuously available in the S's home cage.

\footnotetext{
Apparatus

Rats were tested in a standard operant conditioning chamber with interior dimensions of $22.2 \times 19.1 \times 19.1 \mathrm{~cm}$. The front wall of the chamber had the following configuration: A response lever $3.2 \mathrm{~cm}$ to the right of the center and $6.4 \mathrm{~cm}$ above the grid floor, an opening for access to a liquid dipper $4.4 \mathrm{~cm}$ from the left wall and $2.0 \mathrm{~cm}$ above the floor, a food-pellet cup located $3.0 \mathrm{~cm}$ from the right wall and $3.0 \mathrm{~cm}$ above the floor, and a pilot light located $14.0 \mathrm{~cm}$ above the liquid-dipper opening. The
} 
chamber was placed in a ventilated sound-attenuating ice chest. White noise was always present in the experimental room to mask extraneous noises. Electromechanical relay equipment was located in an adjacent control room and controlled stimulus events and response recording.

\section{Procedure}

During preliminary training, the response lever was retracted. Ss were given five 30-min sessions of dipper training. The dipper contained $0.5 \mathrm{cc}$ of a $10 \%$ sucrose solution (by weight) and was available on a variable-time 1-min (VT 1-min) schedule. Following dipper training, four 30-min sessions of foodcup training were conducted. A $45-\mathrm{mg}$ food pellet was delivered into the food cup on a VT 1-min schedule.

During the next phase of training, the lever remained retracted and sucrose was no longer presented. At this time the Ss were divided into three groups of six each. Ten sessions were conducted in which the Ss were exposed to six $2 \mathrm{~min}$ long light CSs. The mean interval between CSs was $6.5 \mathrm{~min}$. For the $100 \%$ CS-US groups the CS always terminated with the delivery of the food pellet US. For the $66 \%$ CS-US group the CS ended with the delivery of a food pellet on four of the six CS presentations during each session. For the 33\% CS-US group the CS terminated with the delivery of a food pellet on two of the six CSs during a session. The order of CS-US pairings and the CS alone trials were changed every session for the latter two partial-pairing groups.

After these 10 sessions the bar was introduced and remained in the test chamber for the rest of the experiment. Neither the CS nor the US was presented during the next 12 sessions while Ss were conditioned to barpress for sucrose reinforcement. During the first two of these 12 sessions each barpress was reinforced with $6 \mathrm{sec}$ of access to the sucrose solution. For two additional sessions barpresses were reinforced on a VI 45-sec schedule. Finally, for the eight remaining sessions barpresses were reinforced on a VI $90-\mathrm{sec}$ schedule during each 50 -min session.

For the next phase of the experiment the CS-US contingency was introduced while the rats responsed for reinforcement on a VI 90-sec schedule. As in the initial pairing phase six CSs were presented in a 50 -min session with a mean of 6.5 min between each CS. Also, as in the initial conditioning phase, for the $100 \%$ CS-US groups the CS terminated with the delivery of the US on four of the six and two of the six presentations, respectively. This procedure of superimposing the CS-US contingency while the rats were barpressing was followed for 24 sessions with a 2-min CS duration. During the next 36 sessions, the CS duration was changed to $8 \mathrm{sec}$. All other aspects of the procedure remained unchanged.

Inflection ratios were calculated for each $\mathrm{S}$ in each session. The inflection ratio was defined as the number of responses during the six CSs divided by the sum of these CS responses plus the number of responses during a period of time equal to and just preceding the CS. An inflection ratio greater than 0.50 meant that the Ss were responding at a higher rate during the CS than during the immediately preceding interval and was considered evidence of conditioned enhancement. An inflection ratio less than 0.50 meant that Ss were responding at a lower rate during the $\mathrm{CS}$ than during the immediately preceding interval and was evidence of conditioned suppression.

\section{RESULTS}

Figure 1 shows the mean inflection ratios of each group of blocks of four sessions during the last 60 sessions (Blocks 1-15) of the experiment. An analysis of variance was performed on the mean inflection ratios over the first six blocks of sessions in which CS duration

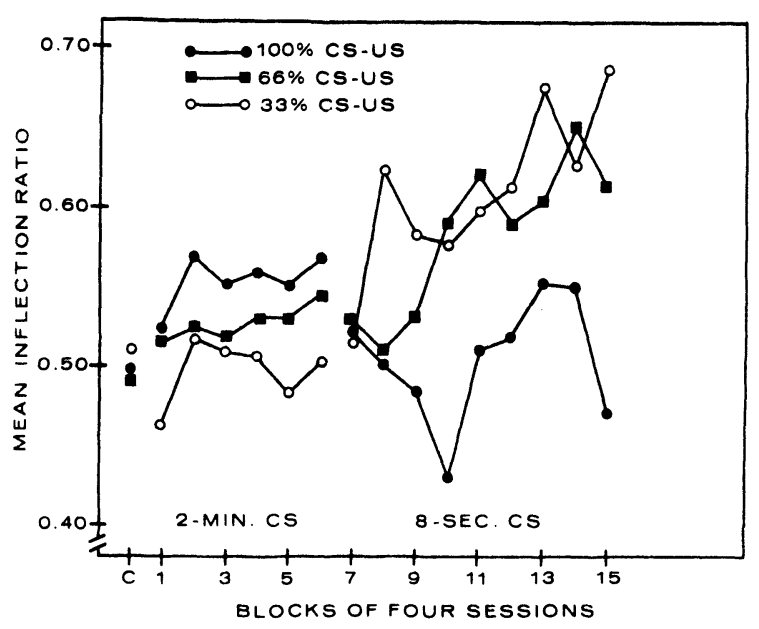

Fig. 1. Inflection ratios as a function of blocks of four sessions for the three different percentages of CS-US pairings. Inflection ratios greater than $\mathbf{5 0}$ represent response enhancement during the CS. The "C" represents the sham inflection ratio when responses were recorded during the pre-CS and CS intervals when no CSs and USs were presented.

was $2 \mathrm{~min}$. The analysis yielded a significant effect of groups $(F=27.11$, df $=2 / 15, p<.01)$. There was also a tendency for the inflection ratios to increase over blocks of sessions $(F=3.20, \mathrm{df}=5 / 75, \mathrm{p}<.02)$. The interaction between groups and blocks of sessions was not significant. To analyze differences between groups, Tukey HSD tests were performed. These tests revealed that the $100 \%$ CS-US group's inflection ratios were greater than both partial pairing groups $(\mathrm{p}<.01$ for both comparisons). Similarly, the $66 \%$ CS-US group's inflection ratios were higher than the $33 \%$ CS-US group's ratios $(\mathrm{p}<.01)$.

In order to determine if a group's inflection ratios were different from $0.50, t$ tests were performed on the difference between the group's mean inflection ratio for the last three blocks and 0.50 . Only the $100 \%$ and $66 \%$ CS-US groups had inflection ratios significantly above $0.50(\mathrm{t}=8.17, \mathrm{p}<.01$ and $\mathrm{t}=4.93, \mathrm{p}<.05$, respectively).

The mean response rate (responses/min) during the 2 min preceding the CS (pre-CS rate) over the six blocks of sessions with a 2-min CS was 17.3 for the $100 \%$ CS-US group. The pre-CS rate for the $66 \% \mathrm{CS}-\mathrm{US}$ and $33 \%$ CS-US groups was 20.0 and 15.1 responses $/ \mathrm{min}$, respectively. An analysis of variance of the asymptotic mean pre-CS response rates over the last three blocks (Blocks 4-6) yielded no significant differences. The mean response rate (responses/min) during the 2 -min CS (CS rate) was 22.1 for the $100 \%$ CS-US group, 23.0 for the $66 \%$ CS-US group, and 15.8 for the $33 \% \mathrm{CS}-$ US group. An analysis of variance of the CS response rate over the last three blocks with a 2-min CS yielded no significant differences.

The analysis of the 8-sec CS stage of the experiment was complicated by the groups entering this stage with 
significantly different inflection ratios and by the increase in the variance of the inflection ratios when the CS duration was changed. Therefore, a separate analysis of variance was preformed for this stage of the experiment on the differences between the S's mean inflection ration during the blocks (four sessions per block) with a 8-sec CS and the same S's mean inflection ratio during the last block of the 2-min CS. An analysis of variance of the change between the 8-sec CS's inflection ratio and the last block of 2-min CS's inflection ratio showed that the difference between groups was significant $(\mathrm{F}=5.56, \mathrm{df}=2 / 15, \mathrm{p}<.05)$. There was also a significant change in inflection ratio as a function of blocks of sessions $(\mathrm{F}=3.10, \mathrm{df}=8 / 120$, $\mathrm{p}<.01$ ) which can be seen as a general increasing trend of inflection ratios for the $66 \%$ and $33 \%$ CS-US groups. The $100 \%$ CS-US group did not show such an increasing trend but the interaction between groups and blocks was not significant. Again to examine differences between groups, Tukey HSD tests were conducted. The $100 \%$ group had an inflection ratio significantly lower than the $66 \%$ group $(p<.01)$ or the $33 \%$ group $(p<.01)$. The $66 \%$ group had a change in inflection ratio which was significantly less than the $100 \%$ group. With a CS of $8 \mathrm{sec}$ the $33 \%$ and $66 \%$ CS-US groups had inflection ratios greater than 0.50 for the last three blocks of sessions $(\mathrm{t}=5.86, \mathrm{p}<.05$ and $\mathrm{t}=8.16, \mathrm{p}<.01$, respectively).

The mean pre-CS response rate (responses/min) for the nine blocks of sessions with a 8-sec CS was 18.6 for $100 \%$ CS-US group, 25.8 for the $66 \%$ CS-US group, and 16.2 responses/min for the $33 \%$ CS-US group. An analysis of variance of the pre-CS response rate over the last three blocks of sessions with a 8-sec CS (Blocks 13-15) yielded no significant differences. The mean CS response rate over the nine blocks of sessions with a 8-sec CS was 27.8 for the $100 \%$ CS-US group, 34.8 for the $66 \%$ CS-US group, and 26.4 for the 33\% CS-US group. Again, an analysis of variance of CS response rates over Blocks 13-15 revealed no significant differences.

T tests were also performed to examine what effects the change in CS duration had on each group's inflection ratios. Here the mean inflection ratios during the last three blocks of sessions with a 2-min CS was compared with the mean inflection ratio during the last three blocks of sessions with an 8-sec CS. The results of these tests showed that the $33 \%(\mathrm{t}=102.5, \mathrm{p}<.001)$ and $66 \%$ CS-US groups $(\mathrm{t}=281.6, \mathrm{p}<.001)$ both had significantly higher inflection ratios when the CS duration was 8 -sec while the $100 \%$ CS-US group had significantly lower $(\mathrm{t}=3.07, \mathrm{p}<.05)$ inflection ratios with an 8-sec CS duration.

\section{DISCUSSION}

The results which were observed in sessions in which long CSs were used are consistent with the type of analysis suggested by two-factor theory (e.g., Rescorla \& Solomon, 1967). The CS could be viewed as eliciting a mediating response compatible with barpressing and thereby causing an increase in response rate. The intensity of this mediating response is probably a function of US probability and, consequently, the degree of conditioned enhancement should be directly related to US probability.

However, this relatively simple interpretation is not very satisfactory when one tries to explain the effects of US probability on response rate during short CS durations. It could be argued that during short CSs the $S$ acquired a mediating response that interferes with the barpress response. This might happen for two different reasons. Azrin and Hake (1969) suggested that CSs which preceded either a positive or negative US elicited an emotional response which would suppress operant behavior. Van Dyne (1971) suggested that CSs preceding positive USs and CSs preceding negative USs elicited different mediating responses, but that both types of responses could interfer with barpressing. In either case, if the strength of the mediating response were directly related to the probability of US presentation, then reduced US probabilities would be expected to lessen response suppression. However, it would not be expected to produce conditioned enhancement such as was observed in this experiment. On the other hand, if the mediating response during a short CS (which was followed by an US on $33 \%$ or $66 \%$ of the trials) were compatible with the operant response, why would increasing the probability of US presentations to $100 \%$ decrease response rate during the CS?

One possible explanation for the effects of US probability on response rate during both long and short CSs is based on the assumption that Ss are making two different types of responses when the CS is presented. One response which occurs in all situations might best be described as similar to the covert emotional response hypothesized by Azrin and Hake (1969) with the difference that we assume this response would not only be compatible with the operant response but would enhance it. As CS duration decreases, the enhancement of responding would increase, but at shorter CS durations Ss probably also make a second response. This second response may be some kind of subtle postural adjustment made in anticipation of the US but which also interferes with operant behavior. We have not been able to specify this response, but our Ss have generally been much less active during short CSs than during long ones. A similar postural adjustment is probably not made during long CSs either because the Ss cannot accurately predict the time at which the US will be delivered or because the number of reinforcements lost by maintaining this posture would be too great. Similarly, when the US occurs with relatively low probability after the short CS the postural adjustment is usually not reinforced and probably will not be maintained.

Thus this hypothesis predicts that when short CSs are used, a low US probability will leave the covert emotional response acting alone and will produce conditioned enhancement. When US probability is high, the postural adjustment is reinforced and response rate during short CSs is suppressed. When long CS durations are used, there is virtually no postural change and the intensity of the covert emotional response is determined by the probability of US presentations. Conditioned enhancement should then be directly related to US probability. These were the results observed in this experiment.

\section{REFERENCES}

Annau, Z., \& Kamin, L. J. The conditioned emotional response as a function of the intensity of the US. Journal of Comparative \& Physiological Psychology, 1961, 54, 428-432.

Brady, J. V. Motivation-emotion factors and intracranial self-stimulation. In D. E. Sheer (Ed.), Electrical stimulation of the brain. Austin: University of Texas Press, 1961.

Brimer, C. J., \& Dockrill, F. J. Partial reinforcement and the CER. Psychonomic Science, 1966, 5, 185-186.

Estes, W. K., \& Skinner, B. F. Some quantitative properties of 
anxiety, Journal of Experimental Psychology, 1941, 29, 290-400

Geller, I. Conditioned suppression in goldfish as a function of shock-reinforcement schedule. Journal of the Experimental Analysis of Behavior, 1964, 7, 345-349.

Gormezano, I., \& Moore, J. W. Classical conditioning. In M. H. Marx (Ed.), Learning: Processes. The MacMillan Company, London, 1969.

Henton, W. W., \& Brady, J. W. Operant acceleration during a prereward stimulus. Journal of the Experimental Analysis of Behavior, 1970, 13, 205-210.

Herrnstein, R. J., \& Morse, W. H. Some effects of response independent positive reinforcement on maintained operant behavior. Journal of Comparative \& Physiological Psychology, $1957,50,461-467$.

Kamin, L. J. Temporal and intensity characteristics of the conditioned stimulus. In W. F. Prokasy (Ed.), Classical conditioning: A symposium. New York: Appleton-Century-Crofts, 1965.

LoLordo, V. M. Facilitation of food reinforced responding by a signal for response-independent food. Journal of the Experimental Analysis of Behavior, 1971, 15, 49-56.

Meltzer, D., \& Brahlek, J. A. Conditioned suppression and conditioned enhancement with the same positive UCS: an effect of CS duration. Journal of Experimental Analysis of Behavior, 1970, 13, 67-74.

Rescorla, R. A., \& Solomon, R. L. Two-process learning theory: Relationship between Pavlovian conditioning and instrumental learning. Psychological Review, 1967, 64, 151-182.

Ross, L. E. The decremental effects of partial reinforcement during acquisition of the conditioned ey elid response. Journal of Experimental Psychology, 1959, 57, 74-82.

Wagner, A. R., Siegle, S., \& Fein, G. G. Extinction of conditioned fear as a function of percentage reinforcement. Journal of Comparative \& Physiological Psychology, 1967, 63, 160-164.

Willis, R. D. The partial reinforcement of conditioned suppression. Journal of Comparative \& Physiological Psychology, 1969, 68, 289-295.

Willis, R. D., \& Ludin, R. W. Conditioned suppression in the rat as a function of shock reinforcement schedule. Psychonomic Science, 1966, 6, 107-108.

(Received for publication August 2, 1974.) 